\title{
Quality assurance for screening mammography: an international comparison
}

C Klabunde, F Bouchard, S Taplin, A Scharpantgen, R Ballard-Barbash, for the International Breast Cancer Screening Network (IBSN)

\begin{abstract}
Study objective-In 1998, the International Breast Cancer Screening Network (IBSN) sponsored an assessment of quality assurance policies and practices to define their scope for population-based screening mammography programmes
\end{abstract} across IBSN countries.

Design-Analysis of data from a survey designed to assess multiple elements of screening programme quality assurance, including organisation of quality assurance activities, mechanisms for site visits and accreditation, requirements for quality control and data systems, and inclusion of treatment, follow up, and programme evaluation in screening mammography quality assurance activities.

Participants and Setting-IBSN representatives in 23 countries completed a comprehensive questionnaire between May and December 1998.

Main results-Completed questionnaires were obtained from all 23 countries. Responses indicated that countries vary in their approaches to implementing quality assurance, although all monitor components of structure, process, and outcome. Nearly all have in place laws, surveillance mechanisms, or standards for quality assurance. In all countries, quality assurance activities extend beyond the screening mammography examination.

Conclusions-The assessment has enhanced understanding of the organisation of screening mammography programmes across countries, as well as the comparability of screening mammography data. All countries have established mechanisms for assuring the quality of screening mammography in population-based programmes, although these mechanisms vary across countries.

(F Epidemiol Community Health 2001;55:204-212)

Ministere de la Sante, Luxembourg

A Scharpantgen

ISBN

A list of members and collaborators is given at the end of the article

Correspondence to: Dr Klabunde, NCI/DCCPS/ARP, Health Services and Economics Branch, EPN Room 4005, 6130 Executive Boulevard, Bethesda, MD 20892-7344, USA (ck97b@nih.gov)

Accepted for publication 5 October 2000 the women who must undergo additional procedures. Moreover, screening mammography is only one element in a sequence of events that leads to a cancer diagnosis or reassurance that cancer is not present (fig 1). The process begins with identification of the target population and proceeds with the invitation to receive screening, the mammography examination, communication of results, and follow up of abnormal examinations through referral for treatment if necessary. It also is iterative in that non-participants may receive repeat invitations to obtain screening, while eligible participants are invited to undergo repeat screening at regular intervals.

Implementation of population-based screening programmes and recognition of the importance of assuring high quality screening examinations have led to several efforts to develop and promote quality assurance standards for mammography. In the United States, the American College of Radiology (ACR), in collaboration with the American Cancer Society, initiated a voluntary mammography accreditation programme in 1987 that has become a national standard for assuring quality. ${ }^{2}$ In conjunction with this programme, ACR published a set of quality control guidelines for radiologists, radiographers, and physicists engaged in mammography. ${ }^{3}$ Since 1989, several documents promulgating standards for mammography in the United Kingdom have been disseminated, including general quality assurance guidelines as well as guidelines specific to nurses, radiographers, breast surgeons, and radiologists. ${ }^{4-8}$ The European Commission, through its "Europe Against Cancer" programme, sponsored the development of guidelines for quality assurance in screening mammography, initially published in 1993 and updated in $1996 .{ }^{9}$ Australia has published accreditation requirements for the mammography facilities that comprise its national screening programme. ${ }^{10} \mathrm{~A}$ set of quality determinants for the multiple provincial and territorial breast cancer screening programmes in Canada also has been disseminated. ${ }^{11}$

The International Breast Cancer Screening Network (IBSN), a voluntary consortium of 23 countries with population-based breast cancer screening programmes, undertook an assessment of the scope of quality assurance activities for screening mammography across member countries in 1998. The goal of the IBSN is to obtain and analyse data on the policies, administration, and outcomes of population-based breast cancer screening. ${ }^{12}$ The assessment was designed to identify the national or other 


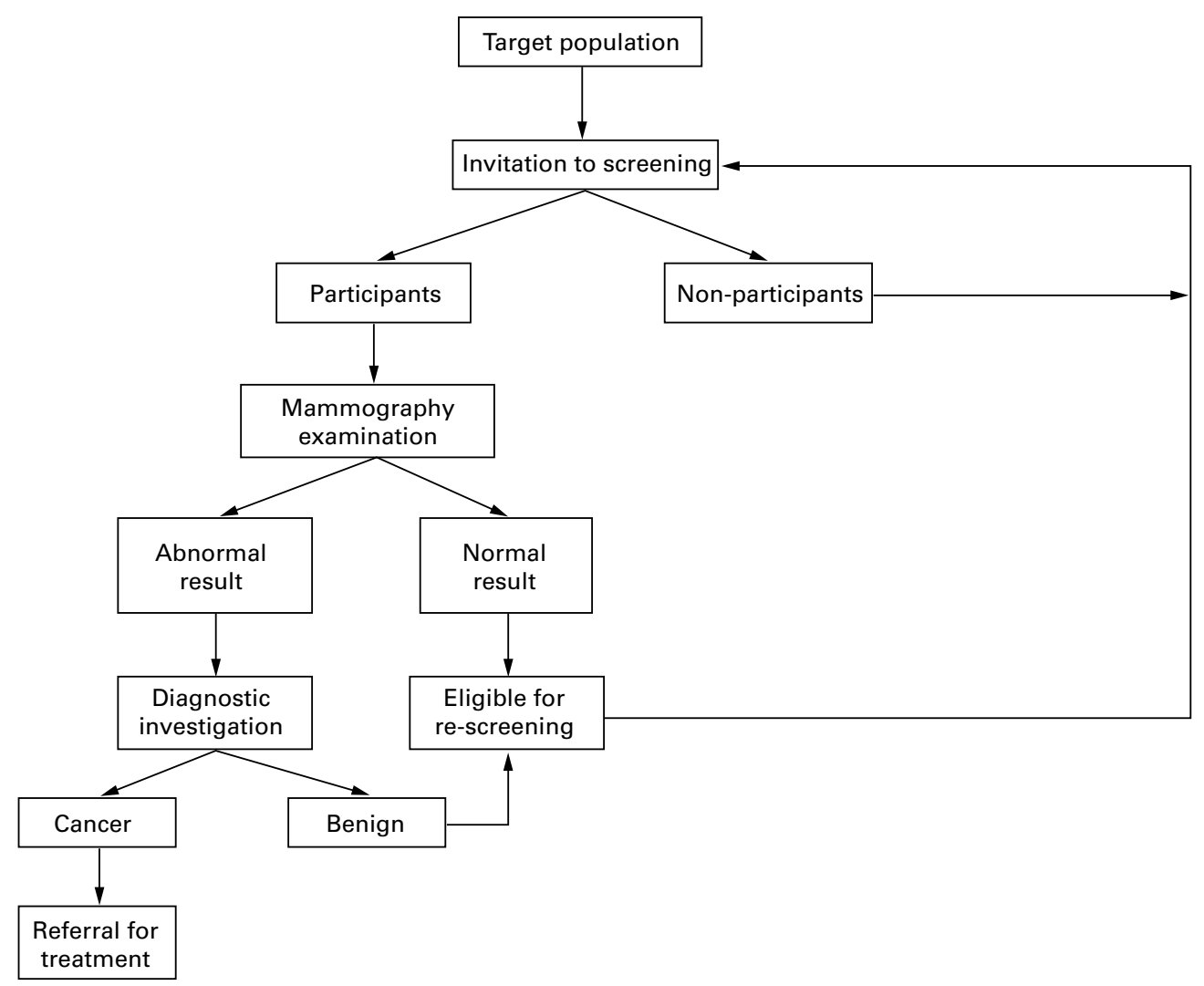

Figure 1 The mammography screening process.

requirements for screening mammography quality assurance that IBSN countries have in place, as well as the specific programmes or activities these countries may have implemented to foster high quality screening mammography performance. This paper provides an international comparison of quality assurance requirements, programmes, and activities for screening mammography programmes, described within the quality assurance domains of structure, process, and outcomes defined by Donabedian. ${ }^{13}$ Donabedian's framework defines quality as encompassing the attributes of the setting in which care takes place, the activities involved in providing care, and the impact of care on health. The paper also describes the external controls that countries may have imposed to ensure a satisfactory level of quality for screening mammography. ${ }^{14}$ The technical quality control, quality assurance in treatment and follow up, and quality assurance for data systems components of the assessment will be summarised in separate papers.

\section{Methods}

An 11 member working group comprised of IBSN representatives from eight countries was formed in October 1997 to develop and implement the quality assurance assessment. ${ }^{\star}$ Be-

*IBSN Quality Assurance Working Group members are: Rachel Ballard-Barbash (NCI, IBSN Chair), Françoise Bouchard (Canada), Mary Codd (Ireland), Andre Grivegnée (Belgium), Edward Hendrick (USA), Carrie Klabunde (NCI, Study Coordinator), Gonzalo Pou (Uruguay), Vitor Rodrigues (Portugal), Hélène Sancho-Garnier (France), Astrid Scharpantgen (Luxembourg), and Stephen Taplin (USA). tween November 1997 and April 1998, the working group constructed a comprehensive questionnaire designed to assess the following aspects of screening programme quality assurance: organisation of quality assurance; site visits and accreditation; quality control; quality assurance for data collection systems; data on treatment, follow up, and mortality; and programme impact. Questionnaire drafts were reviewed by working group members as well as experts in screening mammography and questionnaire design. The finalised questionnaire was mailed in late May 1998 to IBSN representatives in 23 countries. Representatives from countries with organised screening programmes and opportunistic screening (that is, screening occurring outside of organised programmes) were asked to respond for the quality measures of their organised programmes. Representatives from countries with multiple organised screening programmes were asked to provide, if possible, a summary response that reflected the majority of programmes. Because the US does not have a government sponsored organised screening programme comparable to those found in many European and other countries with more centralised health care systems, the questionnaire was sent to a representative from the National Cancer Institute who is responsible for a surveillance research programme focusing on communitybased screening mammography within a multiregion, organised mammography registry system. ${ }^{15}$ Thus, data for the US reflect a diverse subset of health care practices engaged in screening mammography and contributing 
Table 1 Organisation of screening mammography programmes represented in the International Breast Cancer Screening Network, 1998

\begin{tabular}{|c|c|c|c|c|c|c|c|c|}
\hline & $\begin{array}{l}\text { Organised } \\
\text { screening }\end{array}$ & $\begin{array}{l}\text { Year } \\
\text { implemented }\end{array}$ & $\begin{array}{l}\% \text { Target } \\
\text { population }\end{array}$ & $\begin{array}{l}\text { Number of } \\
\text { programmes or } \\
\text { registries }\end{array}$ & $\begin{array}{l}\text { Number of } \\
\text { facilities }^{2}\end{array}$ & $\begin{array}{l}\text { Number of } \\
\text { radiology units }\end{array}$ & $\begin{array}{l}\text { Organisation of } \\
\text { quality assurance }\end{array}$ & $\begin{array}{l}\text { Who finances } \\
\text { quality assurance }\end{array}$ \\
\hline \multicolumn{9}{|l|}{ National programmes } \\
\hline Australia & Yes & 1991 & 54 & 1 & 35 & $35+$ & National & G \\
\hline Finland & Yes & 1986 & 100 & 1 & 11 & 97 & National & $\mathrm{G}, \mathrm{F}$ \\
\hline Iceland & Yes & 1987 & 100 & 1 & 3 & 5 & National & $\mathrm{F}$ \\
\hline Israel & Yes & 1997 & 100 & 1 & 42 & 44 & National & $\mathrm{G}, \mathrm{P}, \mathrm{F}$ \\
\hline Luxembourg & Yes & 1992 & 98 & 1 & 10 & 10 & National & $\mathrm{G}, \mathrm{F}$ \\
\hline Netherlands & Yes & 1989 & 100 & 1 & 55 & 62 & National & G \\
\hline United Kingdom & Yes & 1988 & 100 & 1 & 94 & 315 & National, State & G \\
\hline \multicolumn{9}{|c|}{$\begin{array}{l}\text { State, provincial, local programmes } \\
\text { Established }\end{array}$} \\
\hline Belgium & No & & & 1 & 35 & 40 & Facilities & $\mathrm{G}, \mathrm{F}$ \\
\hline Canada $\ddagger$ & Yes & 1988 & 30 & 10 & 163 & 179 & National, State & $\mathrm{G}$ \\
\hline Denmark & Yes & 1991 & 18 & 1 & 1 & 1 & State & G \\
\hline France & Yes & 1989 & 25 & 26 & 900 & 1000 & National & $\mathrm{G}, \mathrm{P}, \mathrm{F}$ \\
\hline Italył & Yes & 1990 & 10 & 22 & 26 & 43 & State & $\mathrm{G}$ \\
\hline Norway & Yes & 1995 & 40 & 1 & 7 & 15 & National & G \\
\hline Portugal & Yes & 1990 & 20 & 1 & 7 & 7 & State & G \\
\hline Spain & Yes & 1990 & 60 & 1 & 2 & 2 & State & G \\
\hline Sweden $\neq$ & Yes & 1986 & 100 & 26 & 26 & 60 & National & G \\
\hline \multicolumn{9}{|l|}{ Pilot } \\
\hline Germany† & Yes & 1999 & 2 & 3 & 8 & 8 & National, State & $\mathrm{G}, \mathrm{F}, \mathrm{O}^{5}$ \\
\hline Greece & Yes & 1989 & 25 & 3 & 7 & 7 & State & $\mathrm{G}, \mathrm{P}$ \\
\hline Hungary & Yes & 1991 & & 1 & 10 & 10 & State & G \\
\hline Japan & Yes & 1989 & 30 & 1 & 2 & 3 & State & $\mathrm{P}$ \\
\hline Uruguay & Yes & 1996 & 20 & 1 & 1 & 2 & Facilities & $\mathrm{F}$ \\
\hline \multicolumn{9}{|l|}{ Registry programme } \\
\hline United States & No & & & $8 \pi$ & 128 & 166 & National, State & $\mathrm{G}, \mathrm{F}$ \\
\hline
\end{tabular}

$1 \%$ Target population: the proportion of the national population of women deemed eligible to attend for screening and covered by organised screening programmes within the country. ${ }^{2}$ Mammography facility: a location at which women obtain screening mammography. Contains one or more radiography units plus the staff required to perform the procedure. ${ }^{3}$ Organisation of quality assurance: state $=$ state, provincial, local. ${ }^{4} \mathrm{Who}$ finances quality assurance: $\mathrm{G}=$ government, $\mathrm{P}=$ private organisation, $\mathrm{F}=$ mammography facilities, $\mathrm{O}=$ other organisation. ${ }^{5}$ Statutory health funds. $†$ Denotes programme in implementation. $\ddagger$ Denotes countries with a decentralised (subnational) organisation of screening mammography, but in which the totality of organised screening programmes attains national coverage. $\{$ Denotes the number of geographical regions in the US that are participating in the mammography registry programme.

data on mammography practice and outcomes to a centralised statistical centre. Investigators in this mammography registry system are evaluating mammography performance and outcomes within defined populations, an objective common to all of the screening programmes represented in the IBSN. While the facilities providing data to the registry system are not a census of all facilities, they are representative of screening mammography facilities and practice in the US and cover diverse geographical regions in the country.

Completed questionnaires were received from all 23 countries. Data were coded and entered into a Microsoft Access database. Responses were reviewed by the working group in October 1998. After clarification of missing or unclear responses by re-contact of each country's IBSN representative as needed, responses were finalised in December 1998. Because the Republic of Ireland was in the process of developing a new national breast cancer screening programme after concluding a pilot programme, Ireland's response is excluded from this report because of incomplete data.

Comparisons were made between seven countries with population-based screening programmes that are national in scope and 14 countries with screening programmes that are more locally organised. In some cases, these are pilot programmes that eventually may be expanded to encompass a larger geographical area within the country. For example, data provided for Japan reflect the pilot programme in Miyagi prefecture; a national screening mammography programme is being implemented in Japan. Data for Germany are included in this report, although it should be noted that a population-based screening programme was in the planning and implementation stages in Germany at the time of the survey.

It is important to note that nearly all of the countries with organised screening mammography programmes also have opportunistic screening. However, because few of these countries collect or assess data on opportunistic screening, it is impossible to summarise opportunistic screening practice. This assessment reports data obtained from organised screening programmes and registries, the only data currently available for international comparisons of screening mammography practice.

\section{Results}

ORGANISATION OF SCREENING PROGRAMMES AND QUALITY ASSURANCE FOR SCREENING

A broad array of screening mammography programmes are represented in this study (table 1). Of the 22 responding countries, 20 provided data for one or more organised programmes designed to screen a designated target population in a systematic fashion. Programmes vary considerably in year of implementation and in size, indicated by the number of facilities and radiography units for performing screening mammography. National programmes tend to reach a greater proportion of the target population compared with more locally organised programmes. All countries with a national screening programme have implemented quality assurance activities on a national basis. Likewise, most countries with subnational or more locally organised programmes have implemented quality assurance for screening mammography on a subnational 
Table 2 Quality assurance for screening mammography programmes represented in the International Breast Cancer Screening Network: Structural Components, 1998

\begin{tabular}{|c|c|c|c|c|c|c|c|}
\hline & \multirow[b]{2}{*}{$\begin{array}{l}\text { Quality assurance } \\
\text { committee }\end{array}$} & \multirow[b]{2}{*}{ Equipment } & \multirow[b]{2}{*}{$\begin{array}{l}\text { Policies and } \\
\text { procedures }^{I}\end{array}$} & \multicolumn{4}{|c|}{ Personnel requirements ${ }^{2}$} \\
\hline & & & & Licensure & Training & $\begin{array}{l}\text { Minimum number } \\
\text { of examinations }\end{array}$ & $\begin{array}{l}\text { Continuing } \\
\text { education }\end{array}$ \\
\hline \multicolumn{8}{|l|}{ National programmes } \\
\hline Australia & Yes (Biannual) & Yes & $\mathrm{P}, \mathrm{C}, \mathrm{D}, \mathrm{I}$ & $\mathrm{R}, \mathrm{T}, \mathrm{M}, \mathrm{S}$ & $\mathrm{R}, \mathrm{T}, \mathrm{M}, \mathrm{S}$ & $\mathrm{R}, \mathrm{T}$ & $\mathrm{R}, \mathrm{T}$ \\
\hline Finland & No & Yes & P,C,D,I & - & $\mathrm{R}$ & - & - \\
\hline Iceland & No & Yes & $\mathrm{P}, \mathrm{D}, \mathrm{I}$ & - & - & - & $\mathrm{R}, \mathrm{T}$ \\
\hline Israel & Yes (Biannual) & Yes & P,D,I & - & - & - & - \\
\hline Luxembourg & Yes (Monthly) & Yes & $\mathrm{P}, \mathrm{C}, \mathrm{D}, \mathrm{I}$ & - & $\mathrm{T}, \mathrm{M}$ & $\mathrm{T}$ & $\mathrm{T}$ \\
\hline Netherlands & Yes (4-6 times/year) & Yes & $\mathrm{P}, \mathrm{C}, \mathrm{D}, \mathrm{I}$ & $\mathrm{R}, \mathrm{T}$ & $\mathrm{R}, \mathrm{T}$ & $\mathrm{R}$ & $\mathrm{R}, \mathrm{T}$ \\
\hline United Kingdom & Yes (Biannual) & Yes & $\mathrm{P}, \mathrm{C}, \mathrm{D}, \mathrm{I}$ & $\mathrm{T}$ & $\mathrm{T}, \mathrm{M}$ & $\mathrm{R}, \mathrm{T}$ & $\mathrm{R}, \mathrm{T}$ \\
\hline \multirow{2}{*}{\multicolumn{8}{|c|}{$\begin{array}{l}\text { State, provincial, local programmes } \\
\text { Established }\end{array}$}} \\
\hline Belgium & Planned & Yes & P,C,D,I & - & RTM & & \\
\hline Canada $\ddagger$ & Yes (Varies) & Yes & P,C,D & $\mathrm{T}$ & $\mathrm{R}, \mathrm{T}$ & $\overline{\mathrm{R}, \mathrm{T}}$ & $\begin{array}{l}\text { K, } 1 \\
\text { R,T }\end{array}$ \\
\hline Denmark & Yes (6 times/year) & Yes & $\mathrm{P}, \mathrm{C}, \mathrm{D}$ & $\mathrm{R}, \mathrm{T}, \mathrm{M}$ & $\mathrm{R}, \mathrm{T}$ & - & $\mathrm{R}, \mathrm{T}$ \\
\hline France $\ddagger$ & Yes (Quarterly) & Yes & $\mathrm{P}, \mathrm{D}$ & $\mathrm{R}$ & $\mathrm{R}, \mathrm{T}, \mathrm{M}$ & - & $\mathrm{R}$ \\
\hline Italy $\ddagger$ & Yes (Annual) & Yes & $\mathrm{P}, \mathrm{C}, \mathrm{D}, \mathrm{I}$ & - & $\mathrm{R}, \mathrm{T}, \mathrm{S}$ & - & - \\
\hline Norway & Yes (Biannual) & Yes & $\mathrm{P}, \mathrm{C}, \mathrm{D}, \mathrm{I}$ & $\mathrm{S}$ & $\mathrm{R}, \mathrm{T}, \mathrm{S}$ & $\mathrm{R}$ & - \\
\hline Portugal & Yes (Weekly) & Yes & $\mathrm{P}, \mathrm{C}$ & $\mathrm{R}, \mathrm{P}$ & $\mathrm{R}, \mathrm{T}$ & - & $\mathrm{R}, \mathrm{T}$ \\
\hline Spain & Yes (Weekly) & Yes & $\mathrm{P}, \mathrm{C}, \mathrm{D}, \mathrm{I}$ & $\mathrm{S}$ & $\mathrm{R}, \mathrm{T}, \mathrm{M}, \mathrm{S}$ & $\mathrm{R}, \mathrm{T}$ & $\mathrm{R}, \mathrm{T}$ \\
\hline Sweden $\ddagger$ & Yes (Biannual) & Yes & $\mathrm{P}, \mathrm{C}, \mathrm{D}, \mathrm{I}$ & - & $\mathrm{R}, \mathrm{T}$ & $\mathrm{R}$ & - \\
\hline \multicolumn{8}{|l|}{ Pilot } \\
\hline Germany $\dagger$ & Yes (Biannual) & Yes & P,C,D,I & $\mathrm{R}, \mathrm{T}, \mathrm{M}$ & $\mathrm{R}, \mathrm{T}, \mathrm{M}, \mathrm{S}$ & $\mathrm{R}, \mathrm{T}$ & $\mathrm{R}, \mathrm{T}$ \\
\hline Greece & Yes (Quarterly) & Yes & $\mathrm{P}, \mathrm{C}, \mathrm{D}$ & $\mathrm{R}, \mathrm{T}, \mathrm{M}, \mathrm{S}$ & $\mathrm{R}, \mathrm{T}, \mathrm{M}, \mathrm{S}$ & $\mathrm{R}, \mathrm{T}$ & $\mathrm{R}, \mathrm{T}$ \\
\hline Hungary & Yes (Quarterly) & Yes & $\mathrm{P}, \mathrm{C}, \mathrm{D}$ & - & $\mathrm{R}, \mathrm{T}, \mathrm{M}, \mathrm{S}$ & - & $\mathrm{R}$ \\
\hline Japan & Planned & Yes & $\mathrm{P}$ & - & $\mathrm{R}, \mathrm{T}, \mathrm{S}$ & - & - \\
\hline Uruguay & Yes (Monthly) & Yes & $\mathrm{P}, \mathrm{C}, \mathrm{D}$ & $\mathrm{R}, \mathrm{T}$ & $\mathrm{R}, \mathrm{T}$ & $\mathrm{R}, \mathrm{T}$ & $\mathrm{R}, \mathrm{T}$ \\
\hline \multicolumn{8}{|l|}{ Registry programme } \\
\hline United States & Yes $^{3}$ (Varies) & $\mathrm{Yes}^{4}$ & $\mathrm{P}^{4}$ & $\mathrm{R}^{4}, \mathrm{~T}, \mathrm{M}$ & $\mathrm{R}^{4}, \mathrm{~T}, \mathrm{M}$ & $\mathrm{R}^{4}, \mathrm{~T}$ & $\mathrm{R}^{4}, \mathrm{~T}$ \\
\hline
\end{tabular}

${ }^{1}$ Policies and procedures: $\mathrm{P}=$ breast positioning, $\mathrm{C}=$ communication with women about the screening process, $\mathrm{D}=$ minimisation of discomfort, $\mathrm{I}=$ breast implants. ${ }^{2}$ Personnel requirements: $\mathrm{R}=$ radiologist, $\mathrm{T}=$ technologist, $\mathrm{M}=$ medical physicist, $\mathrm{S}=$ surgeon. ${ }^{3}$ Required for quality control components under the Mammography $\mathrm{Quality}$ Standards Act (MOSA) of all mammography facilities in the US. In addition, a National Mammography Quality Assurance Advisory Committee has been established. ${ }^{4}$ Required of all mammography facilities in the US under the Mammography Quality Standards Act. †Denotes programme in implementation. $\ddagger$ Denotes countries with a decentralised (subnational) organisation of screening mammography, but in which the totality of organised screening programmes attains national coverage.

basis. The exceptions are France and Sweden, United Kingdom, Canada, and US, screening which have locally organised screening pro- mammography quality assurance implementagrammes but national requirements for screening mammography quality assurance. Norway, which has national quality assurance requirements, is planning to expand its locally organised programme to a national one. In the tion has occurred on both a national and subnational basis. In two countries, Belgium and Uruguay, quality assurance is the responsibility of the individual mammography facilities in the screening programme.

Table 3 Quality assurance for screening mammography programmes represented in the International Breast Cancer Screening Network: Process Components, 1998

\begin{tabular}{|c|c|c|c|c|c|}
\hline & Recruitment ${ }^{t}$ & Communicate results $^{2}$ & $\begin{array}{l}\text { Mammography } \\
\text { examination }^{3}\end{array}$ & Double reading ${ }^{4}$ & Interpreting/reporting breast lesions \\
\hline \multicolumn{6}{|l|}{ National programmes } \\
\hline Australia & $\mathrm{R}, \mathrm{N}, \mathrm{D}$ & $\mathrm{P}, \mathrm{W}^{6,7,8,9}$ & $\mathrm{Q}, \mathrm{V}, \mathrm{F}$ & All & Standard report, nomenclature \\
\hline Finland & $\mathrm{R}, \mathrm{D}$ & $\mathrm{W}^{6,7,8,9}$ & $\mathrm{Q}, \mathrm{V}$ & All & Standard report \\
\hline Iceland & N,D & $\mathrm{P}, \mathrm{W}^{9}$ & $\mathrm{Q}, \mathrm{V}, \mathrm{F}$ & $95 \%$ & Standard report \\
\hline Israel & $\mathrm{R}, \mathrm{N}, \mathrm{D}^{5}$ & $\mathrm{P}, \mathrm{W}^{5,6,7,8}$ & $\mathrm{Q}, \mathrm{V}$ & Partial & Standard report \\
\hline Luxembourg & $\mathrm{R}, \mathrm{N}, \mathrm{D}$ & $\mathrm{P}, \mathrm{W}^{6,7,8,9}$ & $\mathrm{Q}, \mathrm{V}, \mathrm{F}$ & All & Standard report \\
\hline Netherlands & $\mathrm{R}, \mathrm{N}, \mathrm{D}^{5}$ & $\mathrm{P}, \mathrm{W}^{6,7,8,9}$ & $\mathrm{Q}, \mathrm{V}, \mathrm{F}$ & All & Standard report \\
\hline United Kingdom & R,N,D & $\mathrm{W}^{7,9}$ & $\mathrm{Q}, \mathrm{V}, \mathrm{F}$ & $80 \%$ & Standard report, nomenclature \\
\hline \multicolumn{6}{|c|}{ State, provincial, local programmes } \\
\hline \multicolumn{6}{|c|}{ Established } \\
\hline Belgium & $\mathrm{D}$ & $\mathrm{P}, \mathrm{W}^{6,7,8,9}$ & $\mathrm{Q}, \mathrm{V}, \mathrm{F}$ & All & Standard report, nomenclature \\
\hline Canada $\ddagger$ & $\mathrm{R}, \mathrm{N}, \mathrm{D}$ & $\mathrm{P}, \mathrm{W}^{7,9}$ & $\mathrm{Q}, \mathrm{V}, \mathrm{F}$ & $5-10 \%$ & - \\
\hline Denmark & $\mathrm{R}, \mathrm{N}$ & $\mathrm{W}^{6,7,8,9}$ & $\mathrm{Q}, \mathrm{V}$ & All & Standard report \\
\hline France $\ddagger$ & R,N,D & $\mathrm{P}, \mathrm{W}^{6,7,9}$ & $\mathrm{Q}, \mathrm{V}^{11}$ & All & Standard report \\
\hline Italy $\ddagger$ & R,N,D & $\mathrm{W}^{8}$ & $\mathrm{Q}, \mathrm{V}, \mathrm{F}$ & All & Standard report \\
\hline Norway & R,N,D & $\mathrm{P}, \mathrm{W}^{6,7,8,9}$ & $\mathrm{Q}, \mathrm{V}, \mathrm{F}$ & All & Standard report, nomenclature \\
\hline Portugal & $\mathrm{D}$ & $\mathrm{W}^{6,7,8,9}$ & $\mathrm{Q}, \mathrm{V}, \mathrm{F}$ & All & Standard nomenclature \\
\hline Spain & R,N,D & $\mathrm{P}, \mathrm{W}^{6,7,8,9}$ & $\mathrm{Q}, \mathrm{V}, \mathrm{F}$ & Partial & Standard report \\
\hline Sweden $\ddagger$ & $\mathrm{N}, \mathrm{D}^{5}$ & $\mathrm{~W}^{6,7,8,9}$ & $\mathrm{Q}, \mathrm{V}^{11}$ & All & Standard report \\
\hline \multicolumn{6}{|l|}{ Pilot } \\
\hline Germany† & $\mathrm{R}, \mathrm{N}, \mathrm{D}^{5}$ & $\mathrm{P}, \mathrm{W}^{6,7,8,9}$ & $\mathrm{Q}, \mathrm{V}, \mathrm{F}$ & All & Standard report, nomenclature \\
\hline Greece & $\mathrm{R}, \mathrm{N}, \mathrm{D}^{5}$ & $\mathrm{P}, \mathrm{W}^{6,7,9}$ & $\mathrm{Q}, \mathrm{V}, \mathrm{F}$ & All & Standard report, nomenclature \\
\hline Hungary & $\mathrm{R}, \mathrm{N}$ & $\mathrm{P}, \mathrm{W}^{6,8}$ & $\mathrm{Q}, \mathrm{V}, \mathrm{F}$ & All & Standard report, nomenclature \\
\hline Japan & - & $\mathrm{P}, \mathrm{W}^{6,7,8,9}$ & $\mathrm{Q}, \mathrm{V}^{11}, \mathrm{~F}$ & All & Standard report \\
\hline Uruguay & R,N,D & $\mathrm{P}$ & $\mathrm{Q}, \mathrm{V}, \mathrm{F}$ & $60 \%$ & Standard report, nomenclature \\
\hline \multicolumn{6}{|l|}{ Registry programme } \\
\hline United States & $\mathrm{D}$ & $\mathrm{W}^{6,7,8,9,10}$ & $\mathrm{Q}^{10}, \mathrm{~V}$ & $10-20 \%$ & Standard nomenclature \\
\hline
\end{tabular}

${ }^{1}$ Recruitment: $\mathrm{R}=$ monitor recruitment, $\mathrm{N}=$ monitor reminder procedures for non-participants, $\mathrm{D}=$ collect data on reasons for non-attendance. ${ }^{2}$ Communicate results: $\mathrm{P}=$ to programme staff, $\mathrm{W}=$ to women who undergo screening. ${ }^{3}$ Mammography examination: $\mathrm{Q}=$ film quality, $\mathrm{V}=$ number of views to be taken, $\mathrm{F}=$ feedback on positioning. ${ }^{4}$ Double reading: whether double reading is used for all mammograms, with an estimated proportion of mammograms that are double read in countries that do not always use this procedure. ${ }^{5}$ Routinely. ${ }^{6}$ Receipt/normal results. ${ }^{7}$ Timeliness/normal results. ${ }^{8}$ Receipt/abnormal results. ${ }^{9}$ Timeliness/abnormal results. ${ }^{10}$ Required of all mammography facilities in the US under the Mammography Quality Standards Act (MQSA). ${ }^{11}$ Initial screening mammogram is one view rather than two view. †Denotes programme in implementation. $\neq$ Denotes countries with a decentralised (subnational) organisation of screening mammography, but in which the totality of organised screening programmes attains national coverage. 
Table 4 Quality assurance for screening mammography programmes represented in the International Breast Cancer Screening Network: Outcome Components, 1998

\begin{tabular}{|c|c|c|c|c|c|c|c|c|c|}
\hline & \multicolumn{2}{|c|}{ \% Population screened } & \multicolumn{7}{|c|}{ Monitor/assess } \\
\hline & Set goal & Monitor goal & $\begin{array}{l}\text { Technical } \\
\text { recall }\end{array}$ & $\begin{array}{l}\text { Set maximum } \\
\text { recall }^{\star}\end{array}$ & Follow up care & Adverse events & $\begin{array}{l}\text { Client } \\
\text { satisfaction }\end{array}$ & $\begin{array}{l}\text { Cancer } \\
\text { detection rate }\end{array}$ & $\begin{array}{l}\text { Programme } \\
\text { improvement }\end{array}$ \\
\hline \multicolumn{10}{|l|}{ National programmes } \\
\hline Australia & Yes & Yes & Yes & Yes $(3 \%)$ & Yes & Yes & Yes $^{1}$ & Yes & Yes \\
\hline Finland & No & - & No & - & Yes & Yes & Yes & Yes & Yes \\
\hline Iceland & Yes & Yes & No & - & Yes & No & Yes & Yes & Yes \\
\hline Israel & Yes & Yes & No & - & Yes & Yes & Yes & Yes & Yes $^{1}$ \\
\hline Luxembourg & Yes & Yes & Yes & No & Yes & Yes & Yes & Can be & Yes \\
\hline Netherlands & Yes & Yes & Yes & No & Yes & Yes & Yes & Yes & Yes $^{1}$ \\
\hline United Kingdom & Yes & Yes & Yes & Yes $(3 \%)$ & Yes & Yes & Yes $^{1}$ & Yes & Yes $^{1}$ \\
\hline \multicolumn{10}{|c|}{$\begin{array}{l}\text { State, provincial, local programmes } \\
\text { Established }\end{array}$} \\
\hline Belgium & Yes & No & No & - & Yes & Yes & Yes & Can be & Yes \\
\hline Canada $\ddagger$ & Yes & Yes & Yes & Yes $(2-5 \%)$ & No & No & Yes & Yes & Yes $^{1}$ \\
\hline Denmark & No & - & Yes & No & Yes & Yes & Yes $^{1}$ & Yes & $\mathrm{Yes}^{1}$ \\
\hline France & Yes & Yes & Yes & Yes $(2 \%)$ & Yes & Yes & Yes & Yes & Yes \\
\hline Italy $\ddagger$ & Yes & Yes & Yes & No & Yes & Yes & Yes & Yes & Yes \\
\hline Norway & Yes & Yes & Yes & Yes $(2 \%)$ & Yes & Yes & Yes & Yes & Yes $^{1}$ \\
\hline Portugal & No & - & Yes & Yes $(1 \%)$ & Yes & Yes & Yes & Yes & Yes $^{1}$ \\
\hline Spain & Yes & Yes & Yes & No & Yes & No & Yes & Yes & Yes $^{1}$ \\
\hline $\begin{array}{l}\text { Sweden } \neq \\
\text { Pilot }\end{array}$ & Yes & Yes & Yes & No & Yes & Yes & Yes & Yes & Yes \\
\hline Germany† & Yes & Yes & Yes & Yes $(3-5 \%)$ & Yes & Yes & Yes $^{1}$ & Yes & Yes $^{1}$ \\
\hline Greece & Yes & Yes & Yes & Yes $(3 \%)$ & Yes & Yes & Yes $^{1}$ & Yes & Yes $^{1}$ \\
\hline Hungary & Yes & Yes & Yes & Yes $(5 \%)$ & Yes & Yes & No & Yes & Yes \\
\hline Japan & Yes & Yes & No & - & Yes & No & No & Yes & No \\
\hline Uruguay & No & - & Yes & No & Yes & No & Yes & Yes & Yes $^{1}$ \\
\hline Registry programme & & & & & & & & & \\
\hline United States ${ }^{2}$ & Yes & Yes & Yes & No & $\mathrm{Yes}^{3}$ & $\mathrm{Yes}^{3}$ & Yes $^{1}$ & Yes & Yes \\
\hline
\end{tabular}

${ }^{\star}$ For technical reasons. ${ }^{1}$ Routinely. ${ }^{2}$ The Mammography Quality Standards Act (MQSA) requires all mammography facilities to conduct medical outcomes audits, in which mammography results are systematically collected and compared with outcomes data. Individual facilities determine which outcomes to examine. ${ }^{3}$ Required of all mammography facilities in the US under the Mammography Quality Standards Act (MQSA). †Denotes programme in implementation. $\ddagger$ Denotes countries with a decentralised (subnational) organisation of screening mammography, but in which the totality of organised screening programmes attains national coverage.

STRUCTURAL COMPONENTS

Structural components of quality assurance (table 2) concern whether conditions are conducive to provision of effective care and include requirements for equipment, policies and procedures, and personnel. ${ }^{13}$ Few differences were noted between countries with national and those with more locally organised screening programmes. All countries indicated that quality assurance extends to the equipment used in the mammography exam. Eighteen countries reported having a committee in place to oversee quality assurance activities for the screening programme. Countries were asked whether they had policy and procedure documentation to ensure proper breast positioning, minimisation of discomfort, and proper positioning for women with breast implants during the mammography examination, as well as require that women be informed about all of the steps involved in the examination. All countries reported requiring policy and procedure documentation for breast positioning, while 19 have policies and procedures to ensure minimisation of discomfort during the examination and 17 to facilitate communication with women who undergo screening. Slightly over half of the countries have policy and procedure documentation for women with breast implants.

In 21 countries, quality assurance for screening mammography encompasses the qualifications of the personnel involved in the screening process. Training is the most common requirement, reported by 20 countries, most of which require an initial level of training for the radiographers or technologists who perform screening examinations and for the radiologists who interpret examinations. Nine countries require an initial level of training for physicists and eight initial training for breast surgeons. Thirteen countries specify that radiographers and radiologists keep their skills current through continuing education. Licensure or certification of personnel is a requirement in 13; in these countries, radiographers and radiologists are most often and breast surgeons least often required to be licensed or certified. Twelve countries have standards for performance or interpretation of a minimum number of mammograms by either or both radiographers and radiologists.

\section{PROCESS COMPONENTS}

The process components of quality assurance comprise an assessment of whether the activities involved in providing care are effective. ${ }^{13}$ For screening mammography, process components may involve multiple steps in the mammography sequence (table 3). Nearly all countries reported some form of monitoring to ensure that screening programmes are reaching their intended target population. Sixteen countries indicated monitoring target population recruitment as a quality assurance activity. Seventeen countries reported requiring monitoring of reminder procedures for women who do not accept the invitation to be screened. Although 19 countries indicated that they collect data on reasons for screening nonattendence, two thirds of these reported that they do so only sporadically.

Assessing film quality, specifying the number of views to be taken and whether double reading is to be used, and requiring radiographers to be given feedback by a supervisor on the appropriateness of breast positioning are process components involving the mammography 
Table 5 External controls for quality assurance of screening mammography programmes represented in the International Breast Cancer Screening Network, 1998

\begin{tabular}{|c|c|c|c|c|c|c|}
\hline & \multirow[b]{2}{*}{ Required by law } & \multirow[b]{2}{*}{$\begin{array}{l}\text { Scope of law (year } \\
\text { enacted) }\end{array}$} & \multirow[b]{2}{*}{$\begin{array}{l}\text { Type of screening law } \\
\text { applies to }\end{array}$} & \multicolumn{3}{|c|}{ Mammography facilities } \\
\hline & & & & Have site visits & $\begin{array}{l}\text { Are accredited or use } \\
\text { guidelines }{ }^{4}\end{array}$ & Which guidelines \\
\hline \multicolumn{7}{|l|}{ National programmes } \\
\hline Australia & $\mathrm{No}^{1}$ & & & Yes & Mandatory accred & \\
\hline Finland & Yes & National (1957) & All & Yes & Guidelines & Country specific \\
\hline Iceland & No & & & Yes & Mandatory accred & \\
\hline Israel & No & & & Yes & Mandatory accred & \\
\hline Luxembourg & No & & & $\mathrm{Yes}^{2}$ & Mandatory accred & \\
\hline Netherlands & Yes & National (1997) & Organised & Yes & Mandatory accred & \\
\hline United Kingdom & No & & & Yes & Guidelines & Country specific \\
\hline \multicolumn{7}{|c|}{$\begin{array}{l}\text { State, provincial, local programmes } \\
\text { Established }\end{array}$} \\
\hline Belgium & $\mathrm{No}^{1}$ & & & $\mathrm{Yes}^{2}$ & Guidelines & European \\
\hline Canada $\ddagger$ & No & & & No & Voluntary accred & \\
\hline Denmark & $\mathrm{No}^{1}$ & & & No & Guidelines & European \\
\hline France $\neq$ & Yes & National (1999) & All & $\mathrm{Yes}^{3}$ & Guidelines & Country specific \\
\hline Italy $\ddagger$ & No & & & Yes & Guidelines & European \\
\hline Norway & No & & & Yes & Guidelines & Country specific \\
\hline Portugal & No & & & Yes $^{2}$ & Guidelines & European \\
\hline Spain & Yes & National (1996) & All & $\mathrm{Yes}^{2}$ & Guidelines & European \\
\hline Sweden $\ddagger$ & Yes & National (1990) & Organised & No & Mandatory accred & \\
\hline \multicolumn{7}{|l|}{ Pilot } \\
\hline Germany† & $\mathrm{No}^{1}$ & & & $\mathrm{Yes}^{2}$ & Voluntary accred & \\
\hline Greece & No & & & Yes $^{2}$ & Guidelines & European \\
\hline Hungary & Yes & State (1997) & Organised & Yes & Guidelines & European \\
\hline Japan & No & & & No & No & \\
\hline Uruguay & No & & & Yes & Voluntary accred & \\
\hline \multicolumn{7}{|l|}{ Registry programme } \\
\hline United States & Yes & National (1992) & All & $\mathrm{Yes}^{5}$ & Mandatory accred $^{5}$ & \\
\hline
\end{tabular}

${ }^{1}$ But programme funding is predicated on establishing and maintaining a quality assurance function. ${ }^{2}$ By EUREF. ${ }^{3}$ EUREF site visits for two programmes. Site visits by other organisations for the remainder. ${ }^{4}$ Are accredited or use guidelines: accred $=$ accreditation. ${ }^{5}$ Site visits and accreditation are required of all mammography facilities in the US under the Mammography Quality Standards Act (MQSA). †Denotes programme in implementation. $\ddagger$ Denotes countries with a decentralised (subnational) organisation of screening mammography, but in which the totality of organised screening programmes attains national coverage.

examination. All countries indicated that they specify the number of views to be taken, and require assessment of film quality. Seventeen stipulate that radiographers be given feedback on the quality of breast positioning. In 15 countries, all mammograms are double read, although in three of these countries, only one view is taken at the initial screening examination. Most countries reported facilitating interpretation and reporting of breast lesions either through use of a standardised nomenclature or standardised reports. Nearly all countries monitor communication of mammography results to women who undergo screening.

OUTCOME COMPONENTS

Outcome components of quality assurance concern the extent to which programme objectives are being met, including whether health status is improved as a result of the screening programme. ${ }^{13}$ Thus, assessing outcomes for population-based screening mammography involves activities ranging from monitoring the percentage of the target population that is screened to routine assessment of the cancer detection rate (table 4 ).

All but four countries indicated that they set goals for the percentage of the target population that will be screened. Of the 18 countries that set goals, all but one monitor progress toward achieving those goals. Seventeen countries indicated that they routinely monitor technical recall, or the number of women who are asked to return to the screening unit for a repeat mammogram because of a technical inadequacy in the screening examination; about half of these countries also set and monitor a maximum rate for technical recall. Twenty one countries reported monitoring to ensure that women with abnormal mammograms obtain appropriate follow up care. Three quarters track the occurrence of adverse events such as inaccurate readings and misdiagnoses. Twenty countries generate and assess a cancer detection rate for their screening programmes. Nearly all assess client satisfaction with the screening programme, although fewer $(n=5)$ do so on a routine basis. Similarly, nearly all countries reported that areas for programme improvement are assessed, although only slightly over half do so routinely.

LINKAGE OF STRUCTURE, PROCESS, AND OUTCOME

A comprehensive approach to quality assurance requires elements of all three quality assurance domains: structure, process, and outcome. Attention to all three domains increases the likelihood of quality assurance programme effectiveness because weaknesses in one domain may be balanced by strengths in another. ${ }^{16}$ The data presented in tables 2 to 4 were assessed to determine whether IBSN countries included one or more aspects of each of the domains in the quality assurance activities they reported. In all countries, all three domains are reflected in the screening mammography quality assurance programme.

EXTERNAL CONTROLS FOR QUALITY ASSURANCE External controls for quality assurance involve the laws, surveillance mechanisms, and standards that countries may have implemented for screening mammography. ${ }^{14}$ As shown in table 5 , seven countries have enacted laws that require quality assurance for screening mammography. In six countries these laws are national in scope, and in four they apply to all 
Table 6 Characteristics of site visits to screening mammography programmes in 18 countries, 1998

\begin{tabular}{|c|c|c|c|c|}
\hline & $\begin{array}{l}\text { Site visit } \\
\text { covers }^{1}\end{array}$ & Site visit frequency & Who conducts & Who receives results \\
\hline \multicolumn{5}{|l|}{ National programmes } \\
\hline Australia & $\mathrm{E}, \mathrm{T}, \mathrm{R}, \mathrm{C}, \mathrm{A}, \mathrm{I}$ & Varies & Government, private & Screening programme, mammography facilities \\
\hline Finland & $\mathrm{E}$ & Annual & Government & National Health Authority \\
\hline Iceland & $\mathrm{E}$ & Annual & Government, private & Mammography facilities \\
\hline Israel & $\mathrm{E}$ & Annual & Government & $\begin{array}{l}\text { National Health Authority, screening programme, mammography } \\
\text { facilities }\end{array}$ \\
\hline Luxembourg & $\mathrm{E}, \mathrm{T}^{2}$ & Varies & Screening programme, other & $\begin{array}{l}\text { National Health Authority, screening programme, mammography } \\
\text { facilities }\end{array}$ \\
\hline Netherlands & $\mathrm{E}, \mathrm{T}, \mathrm{R}, \mathrm{C}, \mathrm{A}, \mathrm{I}^{2}$ & Biennial & Other & National Health Authority, screening programme \\
\hline United Kingdom & $\mathrm{E}, \mathrm{T}, \mathrm{R}, \mathrm{C}, \mathrm{A}, \mathrm{I}$ & Every $1-3$ years & Screening programme ${ }^{3}$ & $\begin{array}{l}\text { Local, regional, National Health Authorities; screening programme; } \\
\text { mammography facilities }\end{array}$ \\
\hline \multicolumn{5}{|c|}{$\begin{array}{l}\text { State, provincial, local programmes } \\
\text { Established }\end{array}$} \\
\hline Belgium & $\mathrm{E}, \mathrm{T}^{2}, \mathrm{C}^{2}, \mathrm{~A}^{2}, \mathrm{I}^{2}$ & Biannual & Screening programme & Screening programme \\
\hline France $\ddagger$ & $\mathrm{E}, \mathrm{T}, \mathrm{R}, \mathrm{C}, \mathrm{A}, \mathrm{I}$ & Biannual to annual & Private & National Health Authority, screening programme \\
\hline Italy $\ddagger$ & $\mathrm{E}^{2}$ & Biannual & Screening programme & Screening programme \\
\hline Norway & $\mathrm{E}$ & Annual & $\begin{array}{l}\text { Government, screening } \\
\text { programme }\end{array}$ & Screening programme, mammography facilities \\
\hline Portugal & $\mathrm{E}, \mathrm{T}, \mathrm{C}, \mathrm{A}, \mathrm{I}$ & Biannual & Screening programme & Regional and National Health Authorities, screening programme \\
\hline $\begin{array}{l}\text { Spain } \\
\text { Pilot }\end{array}$ & $\mathrm{E}, \mathrm{T}, \mathrm{R}, \mathrm{C}, \mathrm{A}, \mathrm{I}$ & Biennial & Government & Regional Health Authority, screening programme \\
\hline Germany† & $\mathrm{E}, \mathrm{T}, \mathrm{R}, \mathrm{C}, \mathrm{A}, \mathrm{I}$ & Annual & Screening programme & Screening programme, mammography facilities \\
\hline Greece & $\mathrm{E}, \mathrm{T}, \mathrm{R}, \mathrm{C}, \mathrm{A}, \mathrm{I}$ & Biennial & $\begin{array}{l}\text { Private, screening programme, } \\
\text { other }\end{array}$ & $\begin{array}{l}\text { National Health Authority, screening programme, mammography } \\
\text { facilities }\end{array}$ \\
\hline Hungary & $\mathrm{E}, \mathrm{R}, \mathrm{I}$ & Annual & Screening programme & $\begin{array}{l}\text { National Health Authority, screening programme, mammography } \\
\text { facilities }\end{array}$ \\
\hline $\begin{array}{l}\text { Uruguay } \\
\text { Registry programme }\end{array}$ & $\mathrm{E}, \mathrm{T}, \mathrm{C}, \mathrm{A}^{2}, \mathrm{I}^{2}$ & Biennial & Private & Screening programme, mammography facilities \\
\hline United States $^{4}$ & $\mathrm{E}, \mathrm{T}, \mathrm{C}, \mathrm{A}, \mathrm{I}$ & Annual & Government, private & Regional and National Health Authorities, mammography facilities \\
\hline
\end{tabular}

${ }^{1}$ Site visit covers: $\mathrm{E}=$ equipment, $\mathrm{T}=$ training, $\mathrm{R}=$ recruitment, $\mathrm{C}=$ communication of results, $\mathrm{A}=$ follow up of abnormal results, $\mathrm{I}=$ information systems. ${ }^{2}$ Voluntary. ${ }^{3}$ In the United Kingdom, site visits are conducted by the regional quality assurance team, which is a component of the screening programme. ${ }^{4}$ In the United States, annual site visits are required of all mammography facilities under the federal Mammography Quality Standards Act (MQSA). + Denotes programme in implementation. $\ddagger$ Denotes countries with a decentralised (subnational) organisation of screening mammography, but in which the totality of organised screening programmes attains national coverage.

screening mammography rather than to screening mammography occurring only within organised programmes. Although 15 countries indicated that quality assurance activities are voluntary, programme funding in four of these is predicated on establishing and maintaining a quality assurance function. Over two thirds reported that periodic site visits (inspections of mammography facilities by people who do not work at the screening site) are a required quality assurance component. In seven of these countries, site visits are made by EUREF, a network of reference centres for breast cancer screening established by the "Europe Against Cancer" programme.

Ten countries accredit the mammography facilities that participate in the screening programme; most of the countries that use accreditation have national rather than more locally organised screening programmes. In seven countries, the accreditation is mandatory rather than voluntary. Among the 12 countries that do not have accreditation, all but one (Japan) indicated that facilities are required to follow established guidelines for mammography quality assurance. Seven reported that they

Table 7 Characteristics of accreditation requirements for screening mammography programmes in 10 countries, 1998

\begin{tabular}{|c|c|c|c|c|c|c|c|}
\hline & \multirow[b]{2}{*}{ Administering body } & \multirow[b]{2}{*}{$\begin{array}{l}\text { Frequency of } \\
\text { renewal }\end{array}$} & \multicolumn{5}{|c|}{ Accreditation components } \\
\hline & & & Equipment & Personnel $^{1}$ & $\begin{array}{l}\text { Results } \\
\text { communication }\end{array}$ & $\begin{array}{l}\text { Follow up/ } \\
\text { abnormal results }\end{array}$ & $\begin{array}{l}\text { Information } \\
\text { systems }\end{array}$ \\
\hline \multicolumn{8}{|c|}{ National programmes } \\
\hline Australia & Government agency & Triennial & Yes & $\begin{array}{l}\text { Education: } \mathrm{R}, \mathrm{T} \\
\text { Experience: } \mathrm{R}, \mathrm{T}\end{array}$ & Yes & Yes & Yes \\
\hline Iceland & Government agency & Annual & Yes & No & No & No & No \\
\hline Israel & Government agency & Annual & Yes & No & No & No & Yes \\
\hline Netherlands & Professional association & Annual & Yes & $\begin{array}{l}\text { Education: } \mathrm{R}, \mathrm{T} \\
\text { Experience: } \mathrm{R}, \mathrm{T}\end{array}$ & Yes & Yes & No \\
\hline \multicolumn{8}{|c|}{$\begin{array}{l}\text { State, provincial, local programmes } \\
\text { Established }\end{array}$} \\
\hline Canada & $\begin{array}{l}\text { Professional association, government } \\
\text { agency }\end{array}$ & Triennial & Yes & Education: $\mathrm{R}$ & No & No & No \\
\hline Sweden $\ddagger$ & Professional association & One time & Yes & $\begin{array}{l}\text { Education: R,T,M } \\
\text { Experience: } R, T\end{array}$ & Yes & Yes & Yes \\
\hline $\begin{array}{l}\text { Pilot } \\
\text { Germany }\end{array}$ & Government agency & Triennial & Yes & $\begin{array}{l}\text { Education: R,T,M } \\
\text { Experience: } \mathrm{R}, \mathrm{T}\end{array}$ & Yes & Yes & Yes \\
\hline Uruguay & Private sector organisation & Biennial & Yes & $\begin{array}{l}\text { Education: } \mathrm{R}, \mathrm{T} \\
\text { Experience: } \mathrm{R}, \mathrm{T}\end{array}$ & Yes & Yes & No \\
\hline \multicolumn{7}{|c|}{ Registry programme } & No \\
\hline & agency & & & Experience: $\mathrm{R}, \mathrm{T}$ & & & \\
\hline
\end{tabular}

${ }^{1}$ Personnel: $\mathrm{R}=$ radiologist, $\mathrm{T}=$ technologist (radiographer), $\mathrm{M}=$ medical physicist. ${ }^{2}$ Accreditation is required of all mammography facilities in the US under the Mammography Quality Standards Act (MQSA). †Denotes programme in implementation. ‡Denotes countries with a decentralised (subnational) organisation of screening mammography, but in which the totality of organised screening programmes attains national coverage. 
use the European Guidelines for Quality Assurance in Mammography Screening, ${ }^{9}$ while four (Finland, France, Norway, United Kingdom) have developed country specific guidelines.

Differences in use of external controls are evident between countries with national and those with more locally organised screening programmes. Countries with national programmes are more likely to have enacted legislation mandating quality assurance or to require mandatory accreditation of screening facilities. In contrast, external controls in countries with more locally organised screening programmes are less stringent, with voluntary accreditation of facilities or implementation of quality assurance guidelines the predominant feature.

More detail on the 18 countries that have screening mammography site visit programmes and the 10 countries that require accreditation of screening facilities is provided in tables 6 and 7.

\section{Discussion}

Study results demonstrate that countries with population-based screening programmes have developed a variety of mechanisms to improve the performance and quality of screening mammography. In slightly over half of IBSN countries, quality assurance is implemented on a national basis. Not surprisingly, countries with national screening programmes are more likely to have national requirements for quality assurance than are countries with more locally organised programmes. All countries monitor multiple aspects of quality assurance within the domains of structure, process, and outcome, ${ }^{13}$ and have established linkages across the domains. ${ }^{16}$ The equipment used in the mammography examination is universally monitored. In all countries, quality assurance extends to multiple steps in the mammography screening process, although the breadth of these activities varies.

Although this study shows a comprehensive approach to quality assurance for screening mammography programmes among IBSN countries, a few areas where countries might be able to increase their quality assurance vigilance are evident. For example, some countries indicated that they set goals for target population recruitment or technical recall, but do not have a mechanism for monitoring progress toward attaining the goal. Also, most countries reported monitoring client satisfaction, reasons for nonattendance, and areas for programme improvement, but fewer do so on a routine basis. Furthermore, three countries require double reading of all films, but have implemented a one view rather than two view examination for the initial screen.

External controls for quality assurance range from the legislation that countries may have enacted to requirements for site visits, accreditation, or adherence to guidelines. Countries with national screening mammography programmes seem to have in place more stringent external controls for quality assurance than do countries with more locally organised pro-
KEY POINTS

- Realising the benefit of mammography within population-based screening programmes requires careful attention to the quality of all steps in the screening process.

- In recent years, quality assurance for screening mammography has received increased emphasis internationally.

- Commitment to the key quality assurance domains (structure, process, outcomes) was evident across the 22 countries participating in this study.

- Further work relating specific quality assurance activities to screening mammography performance will increase understanding of how best to achieve the benefits demonstrated in clinical trials.

grammes. This may reflect the greater complexity of managing a programme that is national in scope as well as differing political philosophies within the countries represented. Few other differences in quality assurance activities between countries with national and those with more localised programmes were noted. When compared with a 1995 IBSN programme assessment in which countries with established programmes reported adoption of a greater number of the eight quality assurance components cited in the survey, ${ }^{17}$ current findings suggest an evolution in quality assurance programme development among IBSN countries.

Quality assurance requirements are shaped in part by the approach to screening delivery within a country. For example, in most of the European countries, Canada, and Australia, the screening process begins with identification and recruitment of a target population. European and Canadian guidelines and Australian accreditation requirements therefore include target population recruitment as a central quality assurance component. In contrast, most screening mammography in the US occurs outside the context of an organised programme in which a defined target population is actively recruited; the recommendation to obtain screening usually is made during a doctor-patient visit. As a result, the Mammography Quality Standards Act (MQSA) - which provides a nationwide, uniform framework for mammography quality assurance in the USdoes not address target population recruitment. By identifying broad quality assurance areas, the European guidelines give countries flexibility in interpreting and implementing the requirements, as evidenced by the variations in quality assurance activities noted among the European countries participating in this assessment. The scope of quality assurance activities required by MQSA is not as broad as those outlined in the European guidelines, but implementation is mandatory. Thus, in many IBSN countries there is strong organisation of screening mammography without strict regulation of quality assurance, while in the US screening occurs largely outside of an 
organised context but with legally-based and federally imposed quality assurance requirements.

By documenting the nature and breadth of the quality assurance activities that countries have in place, this assessment has enhanced understanding of the organisation of screening mammography programmes across countries. Moreover, it has afforded insight into the cross national comparability of screening mammography data, and should help inform future IBSN activities such as development of methodologies to evaluate the mortality impact of population-based screening programmes. As an international research consortium, the IBSN is uniquely suited to examining the relation between quality assurance and outcomes within population-based screening mammography programmes. Assessing specific components of screening mammography quality assurance and such performance indicators as participation, compliance, recall, and cancer detection rates and comparing these across programmes are planned activities for this group. Such activities are important to fostering more efficient use of resources, greater understanding of how to achieve the benefits of screening mammography within organised programmes, and improved outcomes for the millions of women worldwide who undergo screening mammography in community settings.

The authors wish to thank Cindy Mattingly and James Cucinell of Information Management Services, Inc, Silver Spring, Maryand, for expert assistance with questionnaire design and database programming, and Freh Kiflemariam of the National Cancer Institute for help with preparation of tables.

IBSN members and collaborators contributing data to the study include: P Tha, B Chapple, Australia; A Grivegnee, Belgium; F Bouchard, Canada; Eynge, Denmark; M Hakama, Finland; H Sancho-Garnier, J Stines, France; L von Karsa, Germany; I Garas, A Linos, E Riza, Greece; E Szabò, A Petrànyi, Hungary; B F Sigfússon, Iceland; J Buttimer, Ireland; G Rennert, Israel; E Paci, E Gentile, M Rosselli del Turco, Italy; N Ohuchi, Japan; A Scharpantgen, Luxembourg; M Broeders, R
Holland, J Hendricks, K Siekman, J Fracheboud, H de Koning, Netherlands; G Skare, Norway; V Rodrigues, Portugal; N Ascunce, Spain; H Malmquist, L Nyström, G Svane, Sweden; S
Moss, J Cooke, J Patnick, United Kingdom; G Pou, Uruguay; R Ballard-Barbash, S Taplin, B Yankaskas, E Hendrick, W Barlow, USA.

Funding: this study was provided with funding by the National Cancer Institute.

Conflicts of interest: none.

1 Bassett LW. Determinants of quality in mammography. Surg Oncol Clin North Am 1997;6:213-32.

2 McLelland R, Hendrick RE, Zinninger MD, et al. The American College of Radiology Mammography Accreditation Program. AfR 1991;157:473-9.

3 Hendrick RE, Bassett LW, Dodd GD, et al. Mammography quality control manuals. Reston, VA: American College of Radiology, 1990, 1992, 1994, 1999

4 National Health Service Breast Screening Programme. Quality assurance guidelines for mammography (Pritchard Report). Sheffield: NHSBSP, 1989.

5 National Health Service Breast Screening Programme. Guidelines for nurses in breast cancer screening. Sheffield: Guidelines for nurses in breast cancer

6 National Health Service Breast Screening Programme. Ouality assurance guidelines for radiographers. Sheffield: Quality assurance guidelines for radi
NHSBSP Publication no 30, 1994.

7 National Health Service Breast Screening Programme. Quality assurance guidelines for surgeons in breast cance screening. Sheffield: NHSBSP Publication no 20, 1996.

8 National Health Service Breast Screening Programme. Quality assurance guidelines for radiologists. Sheffield: NHSBSP Publication no 15, 1997

9 de Wolf CJM, Perry NM, eds. European guidelines for quality assurance in mammography screening. 2nd ed. Luxembourg: European Commission, Europe Against Cancer Programme, 1996.

10 National Program for the Early Detection of Breast Cancer. National accreditation requirements. Canberra: Commonwealth Department of Health and Human Services, 1994.

11 National Committee of the Breast Cancer Screening Initiative. Quality determinants of organized breast cancer screening programs. Ottawa: Health Canada, 1996.

12 Shapiro S, Coleman EA, Broeders M, et al. Breast cancer screening programmes in 22 countries: current policies,
administration, and guidelines. Int $\mathcal{F}$ Epidemiol 1998;27: administrat 42 .

13 Donabedian A. The quality of medical care. Science 1974;200:856-64.

4 Vladeck B. Quality assurance through external controls. Inquiry 1988;25:100-7.

15 Ballard-Barbash R, Taplin SH, Yankaskis BC, et al. Breast cancer surveillance consortium: a national mammography screening and outcomes database. AfR 1997;169:1001-8.

16 Donabedian A. The quality of care: how can it be assessed? $7 A M A 1988 ; 260: 1743-8$.

17 Ballard-Barbash R, Klabunde C, Paci E, et al. Breast cancer screening in 21 countries: delivery of services, notification of results, and outcomes ascertainment. Eur $\mathcal{f}$ Cancer Prev 1999;8:417-26. 